\title{
LAS BECAS 6000 A EXAMEN \\ Resultados, prácticas, expectativas y oportunidades escolares de familias y estudiantes incluidos en el programa
}

\section{EXAMINING BECAS 6000 \\ Scholar performance, practices, expectations and opportunities of families and students included in the program}

\author{
Manuel Ángel Río Ruiz manurio@us.es \\ Universidad de Sevilla. España.
}

\author{
MARÍA LUISA JiMÉNEZ RodRIGO mljimenez@Us.es \\ Universidad de Sevilla. España.
}

\section{RESUMEN}

Este artículo analiza una política de transferencias de rentas condicionadas a resultados educativos entre estudiantes de secundaria postobligatoria de escasos recursos: las Becas 6000 en Andalucía. Se examinan, mediante una revisión de datos administrativos, los resultados escolares del alumnado incluido en el programa. Mediante una estrategia cualitativa, se analizan los efectos de la beca en las estrategias, prácticas y expectativas escolares de diferentes perfiles de becarios y de sus familias. Varios resultados destacan. Primero, aumento del alumnado becado que supera los requisitos académicos exigidos para cobrar la beca. Segundo, existencia de efectos diversos del incentivo en juego en los esfuerzos estudiantiles: mientras que los estudiantes de más alto y de más bajo rendimiento antes de convertirse en becarios apenas modificarían su grado de esfuerzo y compromiso escolar, sí se multiplica entre un importante sector de estudiantes de nivel intermedio. Y, tercero, junto a algunos de los previstos, se dan otros efectos positivos, como la solidificación de las oportunidades escolares futuras del alumnado que consigue finalmente percibir la beca.

\section{Palabras Clave}

Evaluación educativa; Incentivos económicos a la educación; Política educativa; Rendimiento escolar; Transferencias de rentas condicionadas.

\section{Abstract}

This article analyzes a cash-transfer program conditioned to school performance focused on low-income students involved in non-mandatory secondary education: Becas 6000 in Andalusia, Spain. Administrative data on the fellows' achievement are examined and a qualitative research strategy is used to analyze the grant's effects on the schooling strategies, practices and expectations of recipients and their families. Several findings are worth noting. First, the number of fellows who reach academic requirements to receive the grant increases. Second, the incentive at stake produces different results in the efforts of various kinds of students: while those with the highest and the lowest levels of attainment before receiving the grant barely modify their efforts and scholar commitment, middle-range students increase their performance. Third, unexpected positive effects are observed, such as the solidification of future educational opportunities for students who ultimately receive the grant.

\section{KEYWORDS}

Conditional Cash Transfers; Economic Learning Incentives; Education Policy; Educational Evaluation; Scholar Achievement. 


\section{INTRODUCCIÓN}

Desde 2009 Andalucía mantiene un programa de becas limitadas a estudiantes procedentes de hogares con rentas muy bajas. Las Becas 6000 persiguen compensar los costes indirectos de oportunidad derivados de la dedicación plena a estudios no obligatorios, así como incentivar el esfuerzo y rendimiento del alumnado. Para ello, se supedita la percepción de la ayuda formalmente concedida a la demostración continua de resultados escolares a lo largo de los cursos donde esta se ofrece. Además de asistir regularmente, no trabajar ni figurar en desempleo, haber solicitado a la vez la beca compensatoria del gobierno estatal, reunir unos ingresos familiares muy por debajo del umbral de la pobreza; los beneficiarios de Becas 6000 deben aprobar todas las materias en la convocatoria ordinaria de junio, viéndose retenida la ayuda ante cualquier suspenso en las convocatorias trimestrales anteriores ${ }^{1}$.

Las Becas 6000 se articulan como un programa de transferencias de rentas condicionadas a la inversión en capital humano y a la obtención de resultados educativos por parte de sectores desaventajados (Reimers, Da Silva y Trevino 2006; Slavin 2010; Cecchini y Madariaga 2011). Estos programas han sido ampliamente desarrollados en diferentes contextos socioculturales. Son referentes evaluados el Progresa-Oportunidades mexicano, el Bolsa Escola brasileño y el Opportunity NYC estadounidense, orientados principalmente a incentivar la matriculación, asistencia y promoción escolar (Valencia 2008; Riccio et al. 2010). En Europa, destaca el Education Maintenance Allowance (EMA) del Reino Unido (Dearden et al. 2009), basado en pagos semanales para fomentar la asistencia y continuidad de estudiantes de enseñanza postobligatoria. En España, la agenda contra el fracaso y el abandono escolar temprano ha llevado en la última década a programas como las becas-salario para que estudiantes del Programa de Cualificación Profesional Inicial accedieran desde esta vía al último curso de la ESO. Con posterioridad a las Becas 6000 , se han implantado programas similares, aún sin evaluar. Es el caso de otras becas andaluzas, "Segunda Oportunidad", dirigidas a reincorporar al sistema educativo a jóvenes desempleados de baja renta que no concluyeron secundaria obligatoria. Reciben 400 euros mensuales condicionados al progreso satisfactorio en las evaluaciones que se realicen a lo largo del curso. También Extremadura ofrece un incentivo de 1000 euros a jóvenes desempleados a cambio de la obtención del título de ESO y la superación de otras pruebas específicas. Recientemente se ha ampliado este

\footnotetext{
${ }^{1}$ Los ingresos familiares exigidos son la mitad inferiores a los que dan derecho a la beca estatal, concretamente 7306,5€ para un hogar de cuatro miembros; muy por debajo, además, del umbral de la pobreza: $15.768 €$ para el mismo tipo de hogar. La administración andaluza solo inyecta fondos propios -equivalentes aproximadamente a dos tercios de los 6000 euros- entre aquellos estudiantes que cumplan las condiciones académicas de la normativa autonómica. Quienes suspenden alguna materia en junio solo cobrarían entre 2040 y $2429 €$, componente no condicionado correspondiente a la beca estatal compensatoria (Junta de Andalucía 2012).
} 
programa con un complemento de 465 euros para mujeres rurales desempleadas y con cargas familiares que concluyan los estudios secundarios obligatorios.

El análisis multidimensional de un programa pionero como las Becas 6000 representa una oportunidad para evaluar, prácticamente desde el comienzo de estas fórmulas no exentas de polémicas, aspectos cruciales y novedosos de las políticas educativas. Por una parte, el papel de las becas como mecanismo de lucha frente al abandono escolar temprano mediante incentivos a la retención y recuperación de estudiantes desenganchados $^{2}$. Por otra, los efectos de las becas en el rendimiento y las prácticas escolares de la población beneficiaria. Cuestiones estas reactualizadas por las recientes modificaciones ministeriales del sistema de becas que elevan e introducen nuevas exigencias académicas para el acceso, la preservación y la dotación económica de las ayudas ${ }^{3}$. Estos cambios, al menos, están propiciando el debate experto sobre cuáles son los mecanismos más eficientes y equitativos de condicionalidad académica de las becas. Presentamos evidencias empíricas que pueden contribuir a enriquecer ese debate incipiente en el campo de las políticas educativas.

\section{Objetivos y metodología}

El artículo examina los resultados escolares que alcanza la población incluida en el programa Becas 6000. Explica y evalúa los diversos efectos del recurso en juego en las estrategias y prácticas escolares que desarrollan diferentes perfiles académicos de estudiantes becados y sus familias para conservar la ayuda. Por último, muestra las diversas consecuencias de la consecución de la beca sobre las expectativas, decisiones y oportunidades escolares de la población beneficiaria 4 .

Para ello, se analizan datos del registro autonómico de becarios 6000 para los cursos 2009/2010 y 2010/2011 proporcionado por la Dirección General de Participación e Innovación Educativa (DGPIE), y que asciende a 9097 efectivos. Este contiene datos clave sobre la evolución académica del colectivo becado: calificaciones trimestrales y

\footnotetext{
${ }^{2}$ Ayudas enmarcadas dentro de la Estrategia Educación y Formación 2020 para reducir al 10\% el abandono escolar temprano, y que en Andalucía alcanzaba en 2010 un 34,7\% (Ministerio de Educación 2011).

${ }^{3}$ Sin entrar en los nuevos requisitos de las becas universitarias, en secundaria la reforma exige una nota mínima de un 5,5 para poder acceder a una beca en primer curso de Bachillerato y Grado Superior (Gobierno de España 2013). Nuevas condiciones que también repercuten en las Becas 6000 y que han implicado la exclusión de casi 10.000 beneficiarios por no cumplir los nuevos requisitos ministeriales (Junta de Andalucía 2013).

${ }^{4}$ Los resultados proceden de una investigación más amplia -Efectos de las Becas 6000 en las estrategias ante la escolaridad de la población beneficiaria - financiada por el Centro de Estudios Andaluces (convocatoria competitiva 2010/2011). Agradecemos a Esther Márquez Lepe su colaboración en la realización del trabajo de campo para aquel proyecto, donde también se analizaron otros aspectos aquí no abordados como la cobertura, o las necesidades y percepciones sobre la gestión de las ayudas (Río Ruiz y Jiménez Rodrigo 2014; Río Ruiz 2014).
} 
finales registradas en Séneca ${ }^{5}$, promociones y diferencias de rendimiento por tipos de estudio. No se registran, sin embargo, otros datos que hubieran enriquecido el análisis, como los resultados académicos en etapas anteriores de quienes se convierten en becarios. A partir de los datos disponibles hemos podido, no obstante, establecer de cara a la parte cualitativa diferentes tipologías de becarios con desiguales pautas de rendimiento académico tras serle concedida la beca, y con desiguales condiciones de posibilidad de preservarla. Para comparaciones entre becarios y otros estudiantes, se analizan resultados de la Encuesta Social 2010. Educación y hogares en Andalucía, vinculada además al registro Séneca de la Consejería de Educación de Andalucía. Además, se recopilaron datos de estudios y estadísticas sobre becas preuniversitarias y universitarias en Andalucía y España, y se revisaron otras evaluaciones internacionales de programas similares a las Becas 6000 .

Tabla 1.

Perfiles sociodemográficos y académicos del becariado 6000 recogidos en el registro y de los becarios entrevistados.

\begin{tabular}{|c|c|c|c|c|c|}
\hline & \multicolumn{2}{|c|}{ Registro } & \multicolumn{2}{|c|}{ Entrevistas realizadas } \\
\hline & & $\mathrm{n}^{*}$ & $\%$ & $\mathrm{n}$ & $\%$ \\
\hline \multirow{2}{*}{ Sexo } & Mujeres & 3188 & 56,9 & 13 & 52,0 \\
\hline & Hombres & 2414 & 43,1 & 12 & 48,0 \\
\hline \multirow{3}{*}{ Hábitat" } & Menos de 20.000 habitantes & 1379 & 37,3 & 12 & 48,0 \\
\hline & Entre 20.000 y 100.000 habitantes & 925 & 25,0 & 7 & 28,0 \\
\hline & Más de 100.000 habitantes & 1392 & 37,6 & 6 & 24,0 \\
\hline \multirow{2}{*}{ Nacionalidad } & Española & 4662 & 93,0 & 23 & 92,0 \\
\hline & Extranjera & 351 & 7 & 2 & 8,0 \\
\hline \multirow{2}{*}{ Tipos de estudios } & Bachillerato & 3928 & 78,4 & 19 & 76,0 \\
\hline & Grado Medio de Formación Profesional & 1085 & 21,6 & 6 & 24,0 \\
\hline \multirow[b]{2}{*}{ Curso } & Primer curso & 3033 & 60,5 & 8 & 32,0 \\
\hline & Segundo curso & 1980 & 39,5 & 17 & 68,0 \\
\hline \multirow{3}{*}{$\begin{array}{l}\text { Pautas de } \\
\text { rendimiento } \\
\text { académico" }\end{array}$} & $\begin{array}{l}\text { Todas las asignaturas aprobadas } \\
\text { (convocatoria ordinaria) }\end{array}$ & 1525 & 42,0 & 15 & 60,0 \\
\hline & $\begin{array}{l}\text { Una o dos suspensas al final del curso } \\
\text { (convocatoria ordinaria) }\end{array}$ & 709 & 19,5 & 7 & 28,0 \\
\hline & Tres o más suspensas al final del curso & 1398 & 38,5 & 3 & 12,0 \\
\hline
\end{tabular}

"Las mínimas oscilaciones de las $n$ dependen de los cambios en el registro según las altas y bajas en el programa.

"Datos para el curso 2009-2010.

Fuente: Elaboración propia, a partir de los datos del registro sobre becarios 6000 de la Dirección General de Participación e Innovación Educativa.

\footnotetext{
${ }^{5}$ Registro informatizado de la Junta de Andalucía que centraliza datos sobre centros y alumnado de todo el sistema educativo andaluz. Recoge datos, por ejemplo, de calificaciones, asistencia e incidencias disciplinarias de cada estudiante.
} 
A fin de revelar otro tipo de efectos de las becas en las prácticas y decisiones ante la escolaridad se ha realizado un análisis cualitativo de 33 entrevistas en profundidad: 25 individuales a becarios y otras 8 conjuntas a sus madres-padres. La muestra intencional de becarios se estructuró en función de los perfiles académicos y sociales recogidos en el registro administrativo para el curso de realización del trabajo de campo (tabla 1). Otro criterio central de selección fue la diferente trayectoria escolar de los estudiantes antes y después de la concesión de la beca.

Recurrimos para las entrevistas a estrategias de referencia en cadena, y a los propios institutos donde constaban becarios 6000 en casos de perfiles muy específicos. Desarrollamos un guión semiestructurado a partir de los objetivos de investigación, y de las nuevas premisas y categorías emergentes durante el trabajo de campo realizado entre octubre de 2010 y diciembre de 2011. En el curso 2012-13, no obstante, realizamos nuevas entrevistas que permitieron seguir la trayectoria de algunos becarios 6000 posteriormente convertidos en universitarios. Para la organización y codificación de las entrevistas se utilizó el programa ATLAS.ti. El análisis siguió un proceso circular deductivo-inductivo contemplando pautas comunes a la teoría fundamentada, como la comparación constante del corpus cualitativo bajo criterios de saturación.

\section{LOS RENDIMIENTOS DEL BECARIADO EN ESPAÑA}

Los análisis de las becas en España, la mayoría a cargo de economistas de la educación $^{6}$, han dado cuenta de aspectos centrales del sistema, como el gasto público en becas, su alcance y distribución por tipos de hogares (San Segundo 2005; Pérez Esparrels y Vaquero 2009), la contabilización de los costes de oportunidad que las ayudas cubrirían, sus efectos en la demanda educativa y en la continuidad escolar (Calero 1993; De Pablos y Gil Izquierdo 2008), así como la equidad, eficiencia y adecuación de los recursos invertidos (Aldas y Uriel 1999). Mucho menos se han estudiado sus impactos sobre el rendimiento académico (Marcenaro y Navarro 2007; Mediavilla 2010).

Pese a los argumentos esgrimidos para endurecer las condiciones académicas de acceso y preservación de becas, en la universidad los datos disponibles para antes de las reformas recientes muestran mayores aprovechamientos y logros de los becarios, llegándose a diferencias de casi 14 puntos en porcentajes de créditos aprobados (Gráfico 1). Además, los más satisfactorios rendimientos (un $82,5 \%$ de créditos aprobados) se concentran en el umbral 1 , donde se sitúan los becarios-salario, estudiantes de muy escasos recursos económicos (Ministerio de Educación 2011: 13).

\footnotetext{
${ }^{6}$ La economía de la educación se ha limitado únicamente a la cuantificación de una gama limitada de outputs finales de las políticas de becas, un área que necesita de nuevas perspectivas de análisis, como las que supondrían la evaluación cualitativa de programas y reformas educativas (Río Ruiz 2014: Río Ruiz y Jiménez Rodrigo 2014)
} 
Gráfico 1.

Tasa de rendimiento (\% de créditos matriculados-aprobados) del estudiantado universitario general y del becado, según ramas de estudio. Curso 2008-2009.

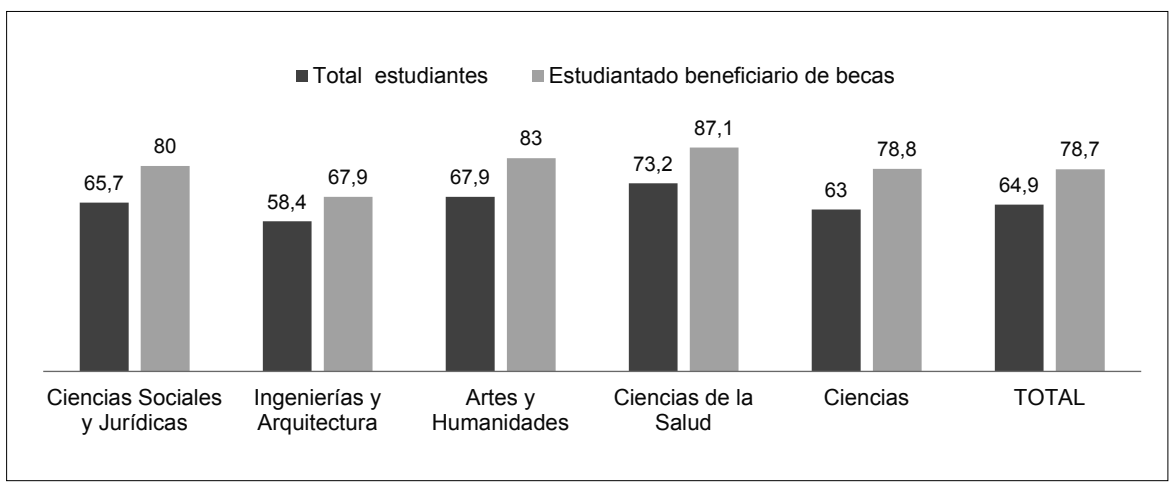

Fuente: Ministerio de Educación, Cultura y Deporte 2012.

Marcenaro y Navarro (2007: 24-25) muestran cómo, además de la obtención de mejores calificaciones entre becarios universitarios, el recurso contribuye a mejorar, en años subsiguientes, el rendimiento de quienes acceden a becas durante el primer curso. Para secundaria postobligatoria, Mediavilla (2010: 573), comparando los rendimientos académicos de dos grupos de estudiantes de características socioeconómicas y edades similares, pero uno perceptor de becas y otro no, constata un efecto positivo de la percepción de becas sobre el nivel educativo logrado a los 19 años, aumentando en un $20 \%$ entre los becarios preuniversitarios la posibilidad de finalizar con éxito y a la edad requerida.

\section{¿Y las Becas 6000? Algunos datos sobre aprovechamientos $Y$ RESULTADOS ESCOLARES}

Las evaluaciones de otros programas similares a las Becas 6000 , muestran poco consistentes impactos de los incentivos económicos sobre los resultados educativos a corto plazo (outputs) de la población incluida (Rawlings y Rubio 2005; Bonal y Tarabini 2006; Reimers et al. 2006; Valencia 2008; Maguire 2008; Slavin 2011; Gneezy et al. 2011). La evaluación del EMA británico apenas identificó mejoras en las calificaciones, salvo entre estudiantes de mayor edad y con rendimientos académicos moderados previos al incentivo (Middleton et al. 2005: 125). En cambio, se observaron mejoras en las puntuaciones de test y exámenes finales de los estudiantes del Opportunity NYC (Riccio et al. 2010: 174). Mayor consenso existe en cuanto a los efectos positivos de estos incentivos sobre 
los inputs de desempeño escolar, como leer determinado número de libros elaborando fichas, incrementar el tiempo invertido en tareas escolares, y la asistencia a clases y tutorías (Legard, Woodfield y White 2001: 53-54; Fryer 2010:38).

En cuanto a las Becas 6000 , se observa en los dos primeros cursos de implantación un incremento interanual del alumnado que consigue alcanzar los objetivos de académicos establecidos por la administración educativa. En dicha evolución se identifican tres situaciones o zonas académicas configuradoras de desiguales posibilidades de mantenimiento de la beca a lo largo del curso escolar (tabla 2).

Tabla 2.

Resultados académicos del becariado 6000.

\begin{tabular}{l|l|c|c|c|c}
\hline \multirow{2}{*}{ Zonas académicas } & \multicolumn{2}{c|}{ Curso 2009/2010 } & \multicolumn{2}{c}{ Curso 2010/2011 } \\
\cline { 3 - 6 } & Resultados académicos & $\mathrm{n}$ & $\%$ & $\mathrm{n}$ & $\%$ \\
\hline Zona de seguridad & $\begin{array}{l}\text { Alumnado que promociona sin } \\
\text { asignaturas pendientes }\end{array}$ & 1.525 & 42,0 & 3.383 & 61,9 \\
\hline $\begin{array}{l}\text { Zona de riesgo moderado } \\
\text { superable }\end{array}$ & $\begin{array}{l}\text { Alumnado que finaliza el curso con } \\
\text { una o dos asignaturas pendientes }\end{array}$ & 709 & 19,5 & 712 & 13 \\
\hline Zona de gran riesgo & $\begin{array}{l}\text { Alumnado que finaliza el curso con } \\
\text { tres o más asignaturas pendientes }\end{array}$ & 1.398 & 38,5 & 1.370 & 25,1 \\
\hline Total & & 3.632 & 100 & 5.465 & 100 \\
\hline
\end{tabular}

Fuente: Elaboración propia, a partir de los datos del registro Becas 6000 de la Dirección General de Participación e Innovación Educativa de la Junta de Andalucía.

Una primera zona "de seguridad" la conforman estudiantes que aprueban todas las asignaturas a lo largo de los trimestres, culminando exitosamente el curso académico en la evaluación final de junio. Este grupo se ha incrementado significativamente con el avance del programa, de forma que en el segundo año seis de cada diez becarios logran conservar la beca. Otra segunda la constituyen estudiantes que pierden la beca por muy escaso margen (zona de "riesgo moderado superable") al suspender una o dos asignaturas en la convocatoria de junio. Muchos de quienes transitan por esta zona consiguen finalmente el objetivo. Otros, aun no consiguiéndolo, pueden volver a solicitarla por segunda vez el próximo curso, por lo que el efecto del incentivo se mantiene de cara al inmediato futuro, pese al revés experimentado al ver la beca "perdida por muy poco" el primer año. Los estudiantes en esta situación han descendido del 19,5\% al 13\%. La tercera zona "de gran riesgo" la integran estudiantes que muestran una elevada acumulación de asignaturas suspensas a lo largo de los trimestres (tres o más) y, en consecuencia, reúnen escasas probabilidades de terminar el curso satisfaciendo el requisito al cual se condiciona la beca. Esta situación es la que más significativamente decrece del $38,5 \%$ al $25 \%$. 
Otro resultado destacable concierne a las buenas calificaciones obtenidas por quienes conservan la beca: el $67 \%$ supera la calificación del notable, siendo el $75 \%$ para bachillerato y el $43 \%$ para ciclos formativos. Ante la ausencia de datos administrativos sobre rendimientos medios globales del estudiantado de postobligatoria, al menos en Andalucía, podemos comparar en el curso 2009/2010 las calificaciones del becariado 6000 con las de otros estudiantes coetáneos en cursos anteriores. En la tabla 3 se observa como las calificaciones de $1 .^{\circ}$ de bachillerato del sector de estudiantes que conservan la beca 6000 supera a las calificaciones obtenidas en un curso menos exigente, $4 .^{\circ}$ de ESO, por una muestra representativa de estudiantes andaluces que, sin repetir ningún curso anteriormente, se matricularon también en bachillerato ese mismo curso. Las calificaciones obtenidas por los perceptores de la beca no solo sobrepasan a las de los estudiantes de su más cercano nivel de ingresos familiares sino también a los de rentas superiores ${ }^{7}$.

Tabla 3.

Calificaciones medias $*^{*}$ obtenidas en $1 .^{\circ}$ de bachillerato por perceptores de Becas 6000 y calificaciones medias obtenidas en $4 .^{\circ}$ de ESO por estudiantes matriculados (sin repetir antes) en $10^{\circ}$ de bachillerato, según nivel de ingresos mensuales familiares. Curso 2009-10.

\begin{tabular}{|c|c|c|c|}
\hline & $\begin{array}{c}\text { Ingresos } \\
\text { familiares } \\
\text { mensuales }\end{array}$ & $\begin{array}{l}\text { Bachillerato } \\
\text { Ciencias } \\
\text { y Tecnología }\end{array}$ & $\begin{array}{c}\text { Bachillerato } \\
\text { Humanidades } \\
\text { Sociales/Artes }\end{array}$ \\
\hline & & \multicolumn{2}{|c|}{ Nota media en $1 .^{\circ}$ Bachilleratos } \\
\hline \multirow[t]{4}{*}{ Becarios 6000 Bachillerato } & $608,9 € 0$ menos $^{* *}$ & $8,05(492)$ & $7,50(642)$ \\
\hline & & \multicolumn{2}{|c|}{ Nota media en $4 .^{\circ}$ de la ESO } \\
\hline & Menos de $1100 €$ & $6,54(117)$ & $5,99(77)$ \\
\hline & De 1101 a $1800 €$ & $7,09(237)$ & $6,16(186)$ \\
\hline \multirow[t]{3}{*}{ Estudiantes $1^{\circ}$ Bachillerato } & De 1801 a $2700 €$ & $6,82(132)$ & $6,18(124)$ \\
\hline & Más de $2700 €$ & $7,30(88)$ & $6,86(110)$ \\
\hline & Total & $7,02(625)$ & $6,21(560)$ \\
\hline
\end{tabular}

" Para estudiantes de $4 .^{\circ}$ de ESO la media se calculó a partir de las calificaciones registradas en Séneca en Matemáticas A-B, Inglés, Lengua Castellana y Literatura y Ciencias Sociales, Geografía e Historia. Para los becarios 6000 se dispone de la media de todas las asignaturas cursadas.

"Calculado en función del umbral anual para acceder a la beca fijado en $7306 €$ anuales para una familia de cuatro miembros.

Fuente: Elaboración propia, a partir de los datos de la Encuesta Social 2010. Hogares y Educación, Instituto de Estadística y Cartografía de Andalucía (IECA) y de los datos del registro sobre Becas 6000 de la Dirección General de Participación e Innovación Educativa.

\footnotetext{
${ }^{7}$ Lo mismo ocurre en grados medios, donde los becarios 6000 obtienen un 6,84 frente al 5,36 en $4 .^{\circ}$ de ESO por parte de los estudiantes matriculados en este tipo de enseñanzas.
} 
Aunque constituya una cuestión crítica, los efectos ilustrados del incentivo en juego sobre el aprovechamiento escolar de los becarios 6000 no pueden valorarse únicamente en función de outputs de rendimiento académico. Otros estudios (Legard et al. 2001; Dobson et al. 2003; Gluz 2006) han subrayado los efectos de estos programas sobre múltiples facetas del desempeño escolar planteando la necesidad de adoptar nuevos enfoques que aprehendan las dinámicas que estos incentivos desencadenan en las percepciones, actitudes y comportamientos de estudiantes y familias participantes. El análisis cualitativo realizado revela una multiplicación de estrategias proescolares y una intensificación de los esfuerzos por parte de un amplio sector de alumnado participante en el programa. Existirian, no obstante, efectos diferenciales en función, fundamentalmente, de las diferentes trayectorias y niveles de rendimiento previo.

\section{Efectos de LAS CONCESIONES de BeCAs 6000 en los ROLES y PRÁcticas ESTUDIANTILES}

No se detecta una pauta común de intensificación del esfuerzo al hilo de la mayor tensión ("un agobio extra") que, en la mayoría de los casos, representaría tener que mantener la beca. Sus efectos dependerán de las diferentes zonas de riesgo académico, variables a lo largo del curso, por las que transitan los becarios 6000 .

\section{Becarios infalibles en zonas de seguridad: alta autoexigencia para mantener el nivel, limitada posibilidad de incremento del esfuerzo, pero más agobio}

Quienes se ubican durante todo el curso por la "zona de seguridad" se movían, ya antes de convertirse en becarios, en altos umbrales de dedicación y autoexigencia escolar. Otro estudio también reveló un efecto escasamente determinativo de estos incentivos como factor intensificador del esfuerzo entre estudiantes de elevado rendimiento, con estrategias estructuradas de estudio, y altas motivaciones escolares basadas en logros previos a su transformación en becarios (Middleton et al. 2005: 121).

El esmero continuo, el cálculo de posibilidades, así como la supeditación de la vida estudiantil a los resultados y al mantenimiento del nivel, forman parte de la identidad de este tipo de estudiantes, acostumbrados a sortear obstáculos y especialmente identificados con las rutinas, exigencias y valores competitivos y meritocráticos de la institución escolar donde depositan todas sus aspiraciones de salvación social ${ }^{8}$. Solo un cataclismo

\footnotetext{
${ }^{8}$ Su perfil encajaría en la semblanza del becario infalible de clase obrera: "Aunque su familia le presione muy poco, con seguridad se presionará a sí mismo más de lo que debería. Empieza a contemplar la vida, tan lejos como pueda visualizarla, como una serie de carrera de vallas, las de las becas que se ganan aprendiendo cómo manejar y acumular la nueva moneda. Tiene la tendencia a sobreestimar la importancia de los exámenes, la acumulación de conocimientos y las opiniones que recibe. Empieza a ver la vida como una escalera, como un
} 
en la depauperada economía familiar ("si veo que no hay nada") o un "tipo de trabajo que no existiría" para ellos - reconocen con desagrado cuando les insistimos en las casuísticas por las que sí abandonarían - les llevaría a abdicar de su rol de estudiante a tiempo completo. El único en el que se proyectan y en el cual quieren seguir identificándose tras haberse convertido en "carne de escuela", que es lo que les "gustaría ser" durante bastante más tiempo. Es el caso de una hija de jornaleros que aspira a mantener sus altas calificaciones para "poder llegar" a medicina:

¿Si yo ahora te digo: "8 horas, 1500 €"? Hombre, lo mismo no estudiaría... Es que, joder: es dinero... ¿Quién te va a pagar por 8 horas $1000 €$, en un día? ¡No!, pero serían $1000 €$ al mes, no al día ¿1000 al mes?... jAh, entonces no! Es que dices: "8 horas, $1000 €$ ” ... ¿1500 $€$ mes y dependienta de una tienda de ropa? $\mathrm{No}$, yo es que quiero estudiar, yo lo que quiero es esto... No. Yo quiero trabajar de lo que a mí me gustaría ser. (Becaria $1,2{ }^{\circ}$ bachillerato).

Mientras que estos estudiantes rechazan deber su abnegación escolar a recompensas extrínsecas, el nivel de autoexigencia mantenida apenas preserva margen de incremento al esfuerzo. No obstante, también se identifican situaciones indicativas de una vivencia estudiantil "algo más agobiada" por la necesidad de conseguir la beca, y por otros factores, como tener que conservar el alto nivel demostrado en nuevos ciclos más exigentes:

Con el cambio de bachillerato, se nota que es más fuerte, pero yo casi siempre sigo en una nota media de 8 más o menos en todos los cursos, que estoy en un nivel fijo. $Y$ ahora, es nivel de bachillerato y saco lo que es mi nota de bachillerato. He bajado un poquillo en inglés. ... Tú rápidamente haces la cuenta, la media, sobre todo el tercer trimestre, que es el más fuerte: "Voy a apretar más, porque son más contenidos, entran más temas en los exámenes, parece que no tienes exámenes, pero la semana que viene los tienes todos juntos". (Becario 12, 1. ${ }^{\circ}$ Bachillerato).

A su vez, las altas expectativas que dominan la vida y la relación con la escolaridad de este tipo de estudiantes también hace difícil discriminar si los mayores agobios y tensiones experimentados se deben per se a la beca:

El día a día, lo notas: “¿Quién lo ha hecho?” Yo sé que me está preguntando a mí. Cuando hacemos un examen, yo sé que está esperando que saque un 80 saque un 9. Es que eso es lo que me pone nerviosa. También porque estoy estudiando y entra mi madre en el cuarto y empieza: "porque tienes que estudiar, porque tu hermana y tu hermano sacaban no sé cuánto..." Entonces, estoy estudiando y estoy pensando: "Tengo que estudiar porque mi hermana sacaba yo que sé qué, tengo que hacer el examen bien, porque el maestro me va a decir no sé cuánto". (Becaria 2, 2. bachillerato).

examen permanente con algunos aplausos y con alguna advertencia en cada escalón" (Hoggart 1970: 347-48). 


\section{Becarios en zonas de gran riesgo: anticipación negativa de oportunidades y res- tricciones en las apuestas escolares}

En el otro extremo, entre los estudiantes en "zona de gran riesgo" de perder la beca, tampoco se identifican dinámicas sostenidas de intensificación del esfuerzo. Las desventajas académicas previamente acumuladas y reproducidas en los nuevos ciclos neutralizarán el efecto positivo del incentivo (Gneezy et al. 2011:197). Ante la continuidad o multiplicación de las malas calificaciones en ciclos más exigentes tiende a desarrollarse un proceso de naturalización temprana de las escasas posibilidades albergadas de cumplimiento final de los (para este tipo de estudiantes) "muy duros" requisitos académicos de la beca. La pérdida anunciada de esta llega en algunos casos al extremo de racionalizarse, en vez de como una oportunidad necesaria desaprovechada, como "un regalo":

La beca estaba para un regalo y era como un plus para estudiar más y, si por una casualidad, yo no quería estudiar, pues... A lo mejor se me dio ese año... me vino cruzado y no. Porque claro, era una clase... En Bachillerato ya se va a estudiar, ¿no?, y se va a estudiar con beca o sin ella, ¿no? (Becario $5,1 .{ }^{\circ}$ bachillerato).

Por otra parte, la acumulación de certezas de pérdida de la beca ("ya que veo que no voy a tener beca") promueve autolimitaciones y modulaciones en las apuestas escolares. Las dudas afloran, por ejemplo, en cuanto a si "ir al final a la universidad"; "buscar un piso en vez de ir y volver"; "hacer el ciclo superior fuera"; seguir "los años que sean" necesarios cuando los padres "a su edad" y "como está la cosa" ya poco van a poder ayudar. A su vez, vías que interferirían en los estudios antes descartadas ("ir a preguntar a ver si hay algo"; "compaginar") comenzarán a barajarse mucho más tras dilapidarse la oportunidad de la beca9:

¿Qué pensé? Pues en soltar el piso, ir y venir y ya está, y ponerme a trabajar si encontraba algo. Cualquier cosa que me viniera... Hombre, si no me viene bien para seguir estudiando, lo dejaría, pero si me dejan venir aquí a dar las clases y tal, pues lo compaginaría. (Becario $9,2{ }^{\circ} \mathrm{GMFP}$ ).

Aunque tenga mis estudios también y sea complicado, pues quiero buscarme también un trabajo y ganar algo más para desahogar algo a mi madre, que tiene ella muchas cosas encima. (Becaria $8,{ }^{\circ}{ }^{\circ}$ bachillerato).

\footnotetext{
${ }^{9}$ Estudios en curso sobre universitarios de clase obrera (Río Ruiz 2014; Jiménez Rodrigo, Río y Caro 2014; Langa y Río 2013) muestran como, al multiplicarse las dificultades de acceso y conservación de becas, se barajan y aplican autolimitaciones con efectos sociales e individuales perversos: regresión a viejas fórmulas autodidactas contraindicadas en el contexto del Plan Bolonia, largos viajes diarios para ahorrar en residencia, y migraciones de estudiantes brillantes hacia titulaciones más baratas y/o donde resultaría menos difícil mantener las becas.
} 
Pese al revés que supone no conseguir la ayuda, los becarios fallidos se declaran dispuestos a "por lo menos terminar" unos estudios hacia los cuales muestran escasa motivación intrínseca. Para muchos la disposición a continuar se debería a la evaporación de las alternativas laborales juveniles que, en cambio, frecuentemente se daban cuando se concibió la beca 6000 - poco antes de la crisis_ como mecanismo de retención escolar del sector juvenil con mayores tasas de abandono en los niveles educativos con mayores riesgos de deserción.

\section{Becarios en zonas de riesgo moderado: remontado el vuelo mediante la multipli- cación de esfuerzos}

En cambio, la más notable intensificación del esfuerzo se produce allí donde la beca se halla en riesgo ante algún suspenso trimestral, pero con posibilidades de recuperación y percepción final. Los estudiantes que marchan por esta zona de "riesgo moderado" son quienes, además de manifestar mayores agobios, demuestran una más nítida adaptación de las rutinas escolares y vitales a las nuevas exigencias académicas asociadas a la beca. El que su concesión sea comunicada habitualmente al final del primer trimestre, así como el cobro en los primeros meses de curso de una parte no condicionada de la beca, contribuye a la confusión y al exceso de confianza de muchos becarios, obligados luego a "remontar el vuelo" y "organizarse bien" tras planear al borde del suspenso y de la consiguiente pérdida de la beca durante alguna fase del curso.

La beca se concibe en estos casos como oportunidad que no se puede desaprovechar y cuya materialización exige someterse a toda clase de privaciones y autocontroles. "Cambio radical", entonces, que reclama también sobreesfuerzos de los padres:

En vez de que mi novio se vaya a las 9:30, que venga a las $8: 30$ y se vaya a las $9, \mathrm{y}$ ya tengo media hora más. Las tardes aprovecharlas más: no ver la tele hasta las 5 , porque a mí me gustan mucho las novelas ... Yo le dije: "Mamá, que voy a cambiar esto radicalmente", porque mi madre me iba a castigar hasta fin de siglo. (Becaria 3 , $1 .^{\circ}$ bachillerato).

La voy a aprobar, es que sí o sí, no me puedo permitir el lujo de suspender. Tengo que hacerlo sobre todo por mí, no me puedo plantear el hacerlo o no hacerlo, no hay otra. ... Yo es que todos los días me acuesto, duermo cinco horas. A eso me acostumbré. (Becaria 14, $2 .{ }^{\circ}$ bachillerato).

"Quiero un profesor particular". Y me dijo mi madre que esperara a las notas y después veríamos. Y yo dije: "Es que no puedo esperar a las notas, es que necesito sacar nota para que no me quede". (Becario 10, 1. ${ }^{\circ}$ bachillerato).

Un asunto controvertido y que concita críticas a estos programas es el de si los esfuerzos para mantener las becas incluyen presiones y negociaciones con el profeso- 
rado. La existencia de alumnos con becas en juego, en vez de valorarse negativamente como situación que pondría en riesgo la igualdad de trato calificador debido a supuestas presiones, podría concebirse en cambio como una oportunidad para que los educadores utilicen las condiciones existenciales de una parte de sus educandos -la presión extra que llegan a sentir quienes se juegan una beca en cada examen-como un recurso más para incentivarlos y mejorar su productividad escolar. Las entrevistas, de hecho, desvelan situaciones donde los profesores, en lugar de sentirse coartados, entendieron la oportunidad que la beca en juego suponía para su labor de subsunción real del contrato educativo frente a habituales negociaciones estudiantiles del esfuerzo (Martín Criado 2010: 273-283) con escasos frutos:

"Tú no me puedes hacer esto, por favor, dame un voto de confianza, apruébame este trimestre". "Es que tú has suspendido". "Vale, hijo, no me des tanta ayuda ni tanto apoyo, que rebosa" ... Tengo una beca, claro que lo he intentado. Y hoy también: "¡Por Dios, que tengo una beca!" $Y$ dice: "Tenías una beca, ¿no?" Cuando me ha dicho cateado con un 4 , por un puto micropunto, me ha entrado de todo. (Becaria $3,1{ }^{\circ}$ bachillerato).

Con o sin implicación del profesorado, en este tipo de estudiantes las estrategias y esfuerzos variados por mantener la beca, obtenga o no finalmente los resultados deseados, promueven cambios positivos en cuanto a las maneras de vivir el rol de estudiante y de afrontar los estudios. Dinámicas que tienden a mantenerse posteriormente (Jackson 2010).

\section{EFECTOS DE LA CONSECUCIÓN FINAL DE LA BECA SOBRE LAS OPORTUNIDADES Y EXPECTATIVAS ESTUDIANTILES}

La consecución final de la beca, además de permitir al estudiante afrontar múltiples inversiones escolares antes limitadas por carencias familiares que promueven procesos de autoexclusión educativa, multiplica las opciones formativas futuras de los estudiantes. Esto se manifiesta sobre todo en los abundantes casos de becarios de zonas rurales, donde la oferta educativa muchas veces es incompleta e implica mayores costes directos a la hora de estudiar. Las Becas 6000 supondrían un importante recurso para amortiguar los efectos discriminatorios de los desequilibrios territoriales que siguen dándose en la provisión de servicios educativos en Andalucía. Gracias a ellas algunos estudiantes de poblaciones rurales alejadas de los núcleos urbanos pueden ampliar sus posibilidades de elección:

Me motivó mucho: "Pues mira, $6000 €$. Pues ya, cuando demos las prácticas en Graná, para el transporte de los autobuses y eso". "Pues mira, ya el autobús no nos tienen que dar nuestros padres tanto, ya nos guardamos, nos administramos para el transporte y ya vamos tirando", porque aquí no hay Grado Superior. (Becario 4, $2{ }^{\circ}$ GMFP). 
Estaba en casa de un familiar, no me querían cobrar nada y a mí me sabía mal, digo: "¿y si me voy a Sevilla y busco piso y un instituto medio qué y acabo?... El que yo vi mejor fue este de cierto renombre (Becario 9, 2. ${ }^{\circ} \mathrm{GMFP}$ ).

La consecución de la beca eleva y afianza las expectativas escolares futuras. El ahorro habitual de parte de lo percibido aumenta la seguridad del estudiante a la hora de proyectarse en el porvenir escolar y de materializar sus apuestas:

A mí se me abrieron las puertas de la universidad, vamos veía la universidad, porque aunque tú digas: "en la universidad también te dan becas". Sí, pero tú, al entrar, tienes que pagar... Entonces, era como tener las espaldas cubiertas de poder empezar a estudiar sin problemas. Yo pensaba un Grado Superior, pero cuando me enteré de que me la habían dado, pues digo: "Bueno, pues a lo mejor tengo oportunidad y puedo estudiar". (Becaria 14, 2. ${ }^{\circ}$ bachillerato).

Con la crisis mi padre se quedó parado ... y entonces si no me hubieran dado la beca 6000 no me hubiera podido venir. Me hubiera tenido que poner a trabajar, porque es que nada más que la matrícula de la universidad vale $1.000 €$ y cómprate materiales, fotocopias. (Becaria 22, $1{ }^{\circ}$ grado Relaciones Laborales).

El hecho de dejar de ser precariamente costeado para convertirse en potencial costeador, si bien por una parte acrecienta el sentimiento de responsabilidad, también contribuye a amortiguar el intenso sentimiento de deuda frente a los sacrificios parentales que experimenta esta clase desaventajada de estudiantes que suele mantener una profunda interiorización del principio de escasez y la conciencia de que las precariedades por las que atraviesan sus familias pueden constituir una seria limitación para poder seguir estudiando. Otros trabajos (Ortí 1982; Langa 2003; Langa y Río Ruiz 2013) han mostrado como entre jóvenes y universitarios de clase obrera el rol de estudiante dependiente de los padres se ejerce inscrito en un sentimiento de deber — de deuda por saldar con la familia- generador de un imperativo moral de restitución que pasa por dejar de ser deudor para convertirse cuanto antes en donante. De forma similar, uno de los elementos centrales de los discursos de los becarios 6000 es el sentimiento de carencia de una familia que pueda mantenerlos indefinidamente y sin dificultades en sus apuestas 0 trayectorias formativas. Frente a lo que revelaron Bourdieu y Passeron (2003) al analizar las actitudes de estudiantes de clase alta, entre estudiantes de extracciones populares rara vez se da una autopercepción de la etapa estudiantil como época para ejercer de diletantes legítimos. Salvo excepciones, como las de estudiantes de familias que han experimentado inesperados procesos de desclasamiento, no subsiste entre los becarios la percepción de la continuidad escolar como derecho no condicionado, inalienable, el cual los padres deberían garantizar independientemente de los resultados escolares obtenidos. Convertirse en aportador gracias a la beca 6000, al tiempo que facilita la apuesta escolar en la familia, contribuye a amortiguar sentimientos de deuda y culpa, 
a legitimar el estatuto improductivo del estudiante a tiempo completo, incluso a mitigar tensiones entre hermanos proletarios aportadores desde que abandonaron tempranamente la escuela y hermanos estudiantes, habitualmente cuestionados por la escasa rentabilidad colectiva de los ejercicios librescos.

Mediante las becas estos jóvenes consiguen mejorar su habitual autopercepción como "tránsfugas de clase" envueltos en matrices de socialización contradictorias -la del universo escolar y la del universo de origen-cuyos valores simbólicos son frecuentemente distintos. Tanto Hoggart (1970: 347) como Lahire (2004: 63) explicaron como los chicos de clase obrera con éxito escolar tienden a transponer los esquemas de valor social incorporados durante la experiencia escolar -la ideología del don, del mérito y del esfuerzo escolar - a sus otros universos como el barrio donde, en cambio, los jóvenes se ven jerarquizados a partir de principios de valor social muy distintos a los que funcionarían en la escuela ${ }^{10}$. En el caso de un becario de etnia gitana se constata esta situación, así como el extraño efecto de la nueva moneda acumulada en la conversión de los "extraños al grupo" en potenciales referentes:

De nuestra raza gitana, pues... vamos, pocos, contados, la mayoría no hace nada, van allí a pasar el tiempo... Y yo creo que eso, que me tienen ahora como referencia, porque incluso allí mi barrio cuando estoy y paso, pues me dicen los niños: "Mira, el de la universidad". "El que va a ir, el que está trabajando, el que ya mismo va a trabajar". (Becario 4, 2. $\left.{ }^{\circ} \mathrm{GMFP}\right)$.

Los sentimientos de deuda mitigados por la beca están a su vez condicionados por el género. La mayoría de las becarias contemplan la ayuda conseguida como medio de resarcir a sus madres, habitualmente valoradas como la parte más sacrificada por el bienestar y la continuidad de los estudios filiales. Es entre becarias de hogares monoparentales donde más visiblemente se manifestará esta actitud solidaria y donde la beca se contemplará también como un recurso individual para no repetir las trayectorias erradas de sus madres, entregadas y absorbidas por proyectos conyugales sin apenas plantearse la posibilidad de "mirar por ellas" cualificándose para la independencia económica frente al varón, posteriormente ausente. Esta experiencia de desamparo, abandono y depauperación femenina incentiva además fenómenos de empoderamiento por parte de las becarias ejemplificados en la frecuente evitación de relaciones afectivas perturbadoras de la dedicación a los estudios:

\footnotetext{
10 "Los becarios desarrollan el sentimiento de no pertenecer ya a ningún grupo. Casi todos los chicos de clase obrera que pasan por el proceso de seguir su educación por medio de becas, durante su adolescencia al menos, tienen roces con su entorno al sentirse desarraigados de su clase: están en el punto de fricción de dos culturas" (Hoggart 1970: 348).
} 
Se lo he dicho muchas veces: "Mamá, déjame que trabaje en algún sitio" "Que no, que no, a estudiar, a estudiar, que no tengas que depender de ningún hombre porque es lo peor que puedes hacer, que te deja tirada y te ves en la calle". El dinero que mi madre me lo administre. Voy a hacer que mi madre lo pueda tocar por encima de todo, se va a empezar a notar en mi casa. (Becaria $3,1{ }^{\circ}$ bachillerato).

\section{EFECTOS DE LA CONCESIÓN DE LA BECA 6000 EN LAS ESTRATEGIAS FAMILIARES DE CONTROL Y ORDENAMIENTO DE LA CONDUCTA ESTUDIANTIL}

La existencia de antecedentes confirmados de buenos resultados escolares hace que se mantenga por parte de los padres la confianza ("ella ya sabe que tiene que esforzarse, porque si no esto no va") en la entrega de los hijos ante el nuevo reto de preservar la beca. En otros casos, donde sí parece intensificarse la presión y el control parental sobre los estudios, no es fácil discriminar si eso se debe principalmente a la existencia de la beca en juego o a otros factores:

Es más presión sobre todo para ella para que estudie, para que no le quede ninguna... "Tienes que poner un poquito más de tu parte". Es que influyen muchas cosas: el no tener que repetir por una o por dos, y la beca... A cada instante me pongo más seria, la castigo, le quito el ordenador. Ya en $2 .^{\circ}$ de Bachiller es más difícil y le cuesta más. También es muy negativa: "yo no soy capaz de hacer esto", "pero chiquilla, ponte". Y luego se pone y lo hace. (Madre de becaria 1, en desempleo, ha trabajado como jornalera y en hostelería).

La presión se incrementará notablemente, en cambio, en los casos y momentos donde algún suspenso trimestral conlleva la retención de la beca. Estas situaciones multiplican las exigencias y medidas parentales para que los menores traduzcan "el agobio" que intrínsecamente suelen experimentar en resultados escolares, no bastando las meras demostraciones de mayor esfuerzo:

Ella está preocupada por la beca, pero también por mí, como yo estoy tan encima en el sentido de que tengo claro que no puedo conseguir dinero por ningún otro sitio, entonces, digo: "Hija, es que date cuenta que es una puerta, hay que compensarlo aprobando, no te queda otra". Yo le tengo bastante control... muchísimo ¡Uf! Algunas veces la agobio, creo que demasiado ... "La de tu hermano la tienes que pagar tú, porque yo ya dentro de 7 años sí que no voy a poder. Y si yo voy a intentar ahora mismo ese dinerito no cogerlo para nada, tú tienes un margen de 7 años, hasta que tu hermano llegue, para estudiar y para trabajar: no te puedes dormir en los laureles". (Madre becaria 3, separada en desempleo). 
Frente al riesgo de perder definitivamente la ayuda, los padres multiplican las demandas de información —ante la administración y los centros o consultando foros de internet- sobre el programa, cuyos novedosos requisitos respecto a otras becas suelen irse conociendo precariamente sobre la marcha. Aunque el papel de las familias ha sido mucho menos considerado, evaluaciones de otros programas subrayan efectos positivos en el desencadenamiento, consolidación y priorización parental de apuestas escolares, especialmente entre familias previamente más distanciadas de las rutinas y normas escolares (Legard et al. 2001: 53).

\section{EFEctos de LA CONSECUCIÓN FILIAL DE LA BECA EN LAS POSIBILIDADES Y APUESTAS ESCOLARES DE LAS FAMILIAS}

La consecución de la beca 6000 tiene también múltiples consecuencias materiales en hogares expuestos a una escasez crónica de recursos. En primer lugar, su llegada alivia las constreñidas economías familiares sin margen para incrementar la inversión escolar, donde buena parte de los progenitores se consideran ya proveedores laboralmente amortizados con escasas posibilidades de generar los recursos necesarios para costear los futuros estudios de sus hijos menores, como sí pudieron hacer con los mayores:

\footnotetext{
¿Qué venderías para que ella siguiera estudiando? MADRE: Ojú, pues vender, como no venda la casa. Hombre, si pudiera, sí ... Y ahora, para ella, todavía más, porque con la mayor éramos más nuevos, trabajábamos... A ver con la chica becaria lo que podemos hacer. Ya somos más viejos y nos cuesta más trabajo. PADRE: Y con la edad que tenemos, con cincuenta y tantos años cada uno. (Padres becaria 1. Madre, jornalera en desempleo. Padre, peón en desempleo).
}

En segundo lugar, la beca permite cubrir justificadas necesidades y demandas filiales no resueltas, como el carné de conducir o la reforma de las habitaciones estudiantiles. A su vez, exonera a los padres de gran parte de los costes escolares cotidianos y permite nuevas inversiones educativas (adquisición de ordenadores y clases particulares, fundamentalmente).

Aunque el cobro de la beca, muchas veces el principal ingreso esperado en estos hogares, suponga un alivio, este no suele conllevar el abandono del principio de escasez que domina las relaciones parentales-filiales. Es más. Ante las hipotéticas expectativas de consumo que podrían albergar los estudiantes al sentirse "dueños de la beca", la percepción de esta se convierte frecuentemente en ocasión para reforzar la vigencia de dicho principio, el cual funcionaría a partir de un contrato de confianza para que los nuevos ingresos sean bien y lealmente administrados por ambas partes: 
Si fuera que va y se compra ella (su madre)... La mataba, porque eso no lo he podido coger yo, que es mío, para comprarme cosas para mí, tampoco... Hombre, si le hiciera falta sí... (Becaria $1,2 .^{\circ}$ bachillerato).

De hecho, la llegada de la beca no suele conllevar recompensas materiales, prevaleciendo una poco voluble sujeción al principio de corresponsabilidad. Al igual que los padres no reciben premios por cumplir sus obligaciones laborales, los menores tampoco pueden esperar recompensas cuando se dedican a cumplir con el oficio de estudiante:

Yo no tengo que premiarla porque haga su trabajo, ese es su trabajo, esa es la obligación que tiene, igual que yo tengo la mía y a mí no me premia nadie. (Madre becaria 3, separada en desempleo).

En tercer lugar, la beca genera posibilidades antes difícilmente contempladas de ahorro familiar para costear gastos de otros miembros del hogar que realizan estudios superiores, muchas veces fuera y sin opción a becas, así como para invertir en los estudios futuros del becario:

Este año han tenido beca ellos dos. Bueno, pero la beca no se les toca, la beca está para la universidad, cada uno tiene su cuenta y la beca se la hemos dejado para la universidad, y los gastos del bachiller salen de nosotros. (Madre becarios gemelos 10, ama de casa).

En los casos donde los padres están algo divididos respecto a las aspiraciones de titulación para el menor, la consecución de la beca contribuye a que se imponga la posición de aquel miembro de la pareja, generalmente la madre, que más elevadas expectativas alberga. Además, la ayuda conseguida actúa como confirmación de las buenas señales escolares de las que depende el mantenimiento de las expectativas educativas de la clase obrera (Terrail 1984), a veces muy frágiles:

PADRE: La vamos a echar al ejército. Ahí sí que gana todos los meses dinero, ahí no falta trabajo, están cobrando todos los meses 1000 euros. MADRE: A aquella [la hermana mayor, también becaria universitaria y estudiante brillante en paro] le decía yo que estudiara una carrera más bajita, que no fuera tan difícil, y ella quiso esa, pues tampoco le voy a decir yo... Yo lo que le digo es que, mientras ella quiera estudiar, yo no la voy a quitar de estudiar. Ahora, que tiene que poner ella de su parte, calentar la silla, no. Yo quiero que estudie, pero... PADRE: Tú te echas uno que tenga bastantes billetes. MADRE: ¡Hombre, pero con sus estudios hechos! (Padres becaria 1. Madre, jornalera en desempleo. Padre, peón en desempleo).

En este tipo de configuraciones familiares prevalece una profunda interiorización de la inflación de los títulos en el mercado de trabajo, algo muchas veces confirmado 
por la experiencia de otros descendientes, familiares o vecinos a quienes de nada sirvieron luego aquellos "carrerones" preferidos. Estas experiencias suelen someter a los padres a un recuento constante de los costes de esfuerzo, de tiempo y de dinero necesario hasta que sus vástagos estén en condiciones de movilizar un título y competir en el mercado laboral. Incluso en casos de estudiantes brillantes, quienes no han ofrecido señales para dudar y limitar las preferencias formativas, los padres suelen decantarse por estudios no muy largos, "no muy difíciles", y con accesos bien delimitados a ocupaciones:

Todo el mundo me decía que era una carrera muy complicada, muy difícil, de dolerle mucho la cabeza ... Y yo le decía: "A. [hermano mayor], ¿por qué no te metes por Magisterio y en tres años tiras la carrera...?" ... Cago en diez, eso lo haces tú de un gorrazo y vas a sacar las oposiciones, y te vas a colocar... ¿Por qué te vas a llevar estudiando tantos años y te vas a partir la cabeza luego, y luego la competencia de los veterinarios, que si pitos, que si flautas?". (Padre de becaria 2, pensionista).

Frente a las más instrumentalistas y continuamente recalculadas apuestas educativas que manifiestan este tipo de padres, obligados por sus constricciones económicas a una continua anticipación negativa de los costes, oportunidades y utilidades de los estudios ante el mercado de trabajo, la conversión en becarios 6000 concede mayor autonomía al estudiante a la hora de optar, aumentando su legitimidad para proyectarse en el porvenir escolar de acuerdo a sus preferencias. Resultado también observado en otros estudios (Escobar y González de la Rocha 2002: 47; Greenberg, Dechausy y Fraker 2011: 6).

\section{CONCLUSIONES}

El examen del registro de becarios 6000 revela un incremento del alumnado que, durante los dos primeros cursos del programa, cumple los requisitos académicos a los que se supedita la percepción de la beca. Además, quienes consiguen percibir la beca obtienen unos resultados académicos superiores a los que exigiría el programa, superando incluso a coetáneos escolares de más elevados ingresos familiares.

El análisis cualitativo ha permitido identificar además otros efectos habitualmente no considerados en las evaluaciones al uso de las políticas de becas, generalmente limitadas a la medición de ciertos outputs de rendimiento académico. La existencia de becas como las examinadas produce efectos que muchas veces no son los formalmente contemplados como objetivos pretendidos por el programa, pero que contribuyen positivamente al alcance de otras metas educativas individuales y colectivas. El análisis también ha permitido profundizar en los efectos diferenciales que la beca genera entre la población incluida. 
Quienes se movían antes de la concesión en umbrales altos de exigencia y rendimiento académico conservan las becas sin dificultades o riesgos, sin afirmar tener que incrementar su ya anteriormente alto esfuerzo. Además, al tiempo que rechazan deber su elevado desempeño a la existencia de recompensas extrínsecas, estos becarios infalibles aparecen firmemente dispuestos a continuar estudiando con o sin beca, si bien son muy conscientes de la contradictoria fragilidad de sus firmes opciones escolares debido a las constricciones económicas de sus familias que compensaría la ayuda. En este sector, la beca también representaría una oportunidad que no podría desaprovecharse y que supone, si no más esfuerzo, sí una fuente de agobio estudiantil extra.

En el otro extremo, quienes acumulaban, antes de convertirse en becarios, trayectorias escolares erráticas que se reproducen en los nuevos y más exigentes ciclos, tampoco intensifican notoriamente su esfuerzo a raíz del nuevo incentivo. Se producen entre estos becarios fallidos procesos de anticipación negativa de oportunidades escolares que naturalizan el fracaso por la pérdida anunciada de la beca. Perderla suele conllevar una rebaja de las apuestas escolares futuras, pero rara vez provoca la decisión de abandonar. Eso no ocurriría debido a la ausencia actual de recambios laborales frente a los estudios, en los cuales se continúa sin mucha motivación intrínseca una vez dilapidadas las posibilidades de obtener recompensas extrínsecas.

En cambio, la intensificación del esfuerzo y los cambios de rutinas hacia la priorización del rol de estudiante, sí que se manifiesta visiblemente entre un amplio sector intermedio de estudiantes con riesgos, pero con posibilidades finales de alcanzar los objetivos académicos para poder percibir la beca. A esto contribuye también el mecanismo - no contemplado, en cambio, en la reforma reciente de las becas estatales-que posibilita la recuperación de la ayuda a lo largo del curso académico y/o al siguiente si se alcanzan las condiciones requeridas. La ayuda, por la cual la mayoría lucha mediante la multiplicación de esfuerzos, privaciones y autocontroles, se viviría también como una oportunidad que no se podría desaprovechar y cuya materialización final se pondría al servicio tanto de necesidades individuales como familiares. Disminuyen así sentimientos de deuda -limitantes de los proyectos educativos - sustentados en la correcta y culpabilizada percepción de que, mientras las inversiones educativas son familiares, los beneficios diferidos son individuales.

Por tanto, además de permitir afrontar constricciones materiales mientras se estudia y neutralizar los frecuentes dilemas entre estudiar y/o aportar, las rentas transferidas permiten solidificar las apuestas escolares futuras de estudiantes y familias. En suma, además de representar un importante recurso para aliviar y saldar cuentas en el presente, como prevé la política, estas becas contribuyen a la proyección con más seguridad en el porvenir escolar.

Aunque serían diversos los efectos positivos de las Becas 6000, en las actuales $-\mathrm{y}$ no previstas cuando se diseñó la política - condiciones de masivo desempleo juvenil y de inesperadas continuidades y regresos escolares por tal motivo, sí podría cuestionarse (en estos momentos) su protagonismo en la retención escolar de jóvenes sin recursos 
familiares mediante la compensación de costes de oportunidad. El examen efectuado plantearía, así, una cuestión poco contemplada al evaluar políticas públicas. Cómo estas pueden tener múltiples efectos positivos, muchas veces no previstos, incluso cuando cambian algunas condiciones sociales y se ve, entonces, dificultado el logro de alguno de los objetivos sí declarados en el momento de concebirlas.

El examen de las Becas 6000 aportaría hallazgos para el debate sobre la eficiencia de estas políticas educativas, pudiendo contribuir a la reformulación del programa y/o al diseño futuro de otras políticas de becas. Sin embargo, el debate no debiera limitarse exclusivamente a cuestiones de eficiencia, como se ha pretendido en recientes reformas del sistema de becas justificadas mediante más adjetivos que datos. Correspondería a la ciudadanía posicionarse políticamente sobre una cuestión crucial que afecta al principio de equidad. La de si es de justicia redistributiva exigir académicamente más para poder optar a becas a una hija de jornalero que a un hijo de una cirujana sí capacitada económicamente para afrontar los costes directos e indirectos de los estudios filiales sin necesidad de ayudas públicas.

\section{REFERENCIAS BibLIOGRÁfICAS}

Aldas, J. y Ezequiel U. 1999. "Equidad y eficacia del sistema español de becas y ayudas al estudio". Working Paper n. ${ }^{\circ}$ EC99-11, Instituto Valenciano de Investigaciones Económicas, Valencia.

Bonal, X. y A. Tarabini. 2006. "Programas de garantía de renta condicionados a la asistencia escolar. Una revisión de evaluaciones del programa Bolsa Escola". Revista Mexicana de Investigación Educativa 11:943-70.

Bourdieu, P. y J. C. Passeron. 2003. Los herederos. Los estudiantes y la cultura. Buenos Aires: Siglo XXI.

Calero, J. 1993. Efectos del gasto público educativo: el sistema de becas universitarias. Barcelona: Publicacions i Edicions de la Universitat de Barcelona.

Cecchini, S. y A. Madariaga. 2011. Programas de transferencias de rentas condicionadas. Balance de la experiencia reciente en América Latina y el Caribe. Santiago de Chile: CEPAL.

Dearden, L., C. Emmerson, C. Frayne y C. Meghir. 2009. "Conditional Cash Transfers and School Dropout Rates". Journal of Human Resources 44: 827-857.

Dobson, B., J. Hardman, S. Maguire, S. Middleton, T. Allen, J. Graham, E. Hill, K. Woodfield y M. Maguire. 2003. Education Maintenance Allowance Pilots for Vulnerable Young People and Childcare Pilots: Implementation and Reported Impacts in the First Two Years (2000-2001/2001-2002). Department for Education and Skills Research Report n. ${ }^{\circ}$ 470. Nottingham: DfES Publications.

Escobar, A. y M. González de la Rocha. 2002. Evaluación cualitativa del Programa de Desarrollo Humano Oportunidades. Seguimiento de impacto 2001-2002: comunidades de 2500 a 50.000 habitantes. Guadalajara: CIESAS Occidente.

Fryer, R. 2010. "Financial Incentives and Student Achievement: Evidence from Randomized Trials". NBER Working Paper n. ${ }^{0}$ 15.898, Department of Economics, Harvard University, Cambridge, MA. 
Gluz, N. 2006. La construcción socioeducativa del becario: la productividad simbólica de las politicas sociales en la educación media. Buenos Aires: Instituto Internacional de Planeamiento de la Educación-Unesco.

Gneezy, U., S. Meier y P. Rey-Bie. 2011. "When and Why Incentives (Don't) Work to Modify Behavior". Journal of Economics Perspectives 4: 191-210.

Gobierno de España. 2013. "Real Decreto 609/2013, de 2 de agosto, por el que se establecen los umbrales de renta y patrimonio familiar y las cuantías de las becas y ayudas al estudio para el curso 2013-2014, y se modifica parcialmente el Real Decreto 1721/2007 por el que se establece el régimen de las becas y ayudas al estudio personalizadas". Boletín Oficial del Estado 185: 5670021. Madrid: BOE.

Greenberg, D., N. Dechausay y C. Fraker. 2011. Learning Together. How Families Responded to Education Incentives in New York City's Conditional Cash Transfer Program. Nueva York: MDRC.

Hoggart, R. 1970. La culture du pauvre. Étude sur le style de vie des classes populaires en Angleterre. París: Minuit.

Jackson, C. K. 2010. "A Little Now for a Lot Later: a Look at Texas Advanced Placement Incentive Program". Journal of Human Resources 45: 591-639.

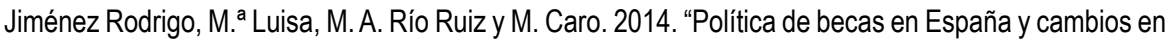
sus condiciones académicas de preservación: las experiencias, dilemas, recálculos y estrategias de un becariado en riesgo". Pp. 97-101 en C. Peláez y M.I. Jociles (eds.) Estudios etnográficos de las políticas públicas en centro educativos. Madrid: Traficantes de Sueños.

Junta de Andalucía. 2012. "Resolución de 6 de julio de 2012 por la que se efectúa la convocatoria pública de la Beca 6000 dirigida a facilitar la permanencia en el sistema educativo del alumnado de bachillerato o de ciclos formativos de grado medio de formación profesional inicial para el curso escolar 2012-2013". Boletín Oficial de la Junta de Andalucía 141: 9-11. Sevilla: BOJA.

Junta de Andalucía. 2013. "La Junta denuncia que el Ministerio de Educación deja sin becas a 10.000 alumnos andaluces". Consulta 20 mayo 2013 (http://www.juntadeandalucia.es/educacion/educacion/nav/contenido.jsp?pag=/Contenidos/GabinetePrensa/Notas_de_prensa/2013/Marzo/nota_ prensa_130313_becas).

Lahire, B. 2004. El hombre plural. Los resortes de la acción. Barcelona: Bellaterra.

Langa, D. 2003. "Los estudiantes y sus razones prácticas: heterogeneidad de estrategias de universitarios según clase social". Tesis doctoral, Departamento de Sociología I, Universidad Complutense de Madrid, Madrid.

Langa, D. y M. A. Río Ruíz. 2013. "Los estudiantes de clases populares en la universidad y frente a la universidad de la crisis: persistencia y nuevas condiciones para la multiplicación de la desigualdad de oportunidades educativas". Témpora, Revista de Sociología de la Educación 16: 71-96.

Legard, R., K. Woodfield y C. White. 2001. "Staying Away or Staying on? A Qualitative Evaluation of the Education Maintenance Allowance". Research report n. ${ }^{\circ}$ 256, Department for Education and Employment. Londres.

Maguire, S. 2008. "Paying Young People to Learn - Does it Work?" Research in Post-Compulsory Education 13: 205-15. 
Marcenaro, Ó. y L. Navarro. 2007. "El éxito en la universidad: una aproximación cuantílica". Revista de Economía Aplicada 44: 5-39.

Martín Criado, E. 2010. La escuela sin funciones. Crítica de la sociología de la educación crítica. Barcelona: Bellaterra.

Mediavilla, M. 2010. "Las becas y ayudas al estudio como elemento determinante de la continuidad escolar en el nivel secundario post-obligatorio. Un análisis de sensibilidad a partir de la aplicación del Propensity Score Matching". Investigaciones de Economía de la Educación 5: 561-82.

Middleton, S., K. Perren, S. Maguire y J. Rennison. 2005. "Evaluation of Education Maintenance Allowance Pilots: Young People Aged 16 to 19 Years". Final Report of the Quantitative Evaluation, Department for Education and Skills Research Report №. 678. Nottingham: DfES Publications.

Ministerio de Educación. 2011. Objetivos educativos europeos y españoles. Estrategia educación y formación 2020. Informe español 2010-2011. Madrid: Ministerio de Educación.

Ministerio de Educación. 2012 y 2013. Secretaría General de Universidades. Datos y cifras del sistema universitario español. Madrid: MECD.

Ortí, A. 1982. "Unidad y diversidad de la mentalidad y actitudes ideológicas juveniles en la post-transición democrática". (Informe inédito mimeografiado).

(De) Pablos, L. y M. Gil Izquierdo. 2008. "Análisis de la incidencia de reformas en el sistema de financiación de la educación universitaria en España a partir de un modelo de comportamiento". Hacienda Pública Española / Revista de Economía Pública 184: 117-52.

Pérez Esparrells, M. ${ }^{a}$ C. y A. Vaquero. 2009. "La financiación de la enseñanza obligatoria en un contexto de cambios demográficos y sociales". Papeles de Economía Española 119: 228-45.

Rawlings, L. y G. Rubio. 2005. "Evaluating the Impact of Conditional Cash Transfer Programs". The World Bank Research Observer 20: 29-55.

Reimers, F., C. Silva y E. Trevino. 2006. Where is the "Education" in Conditional Cash Transfers in Education? Unesco: Montreal.

Riccio, J., N. Dechausay, D. Greenberg, C. Miller, Z. Rucks y N. Verma. 2010. Toward Reduced Poverty across Generations. Early findings from New York City's Conditional Cash Transfer Program. Nueva York: MDRC.

Río Ruíz, M. A. y M. ${ }^{a}$ L. Jiménez Rodrigo. 2012. "Tú pones el esfuerzo, nosotros los recursos: una evaluación sobre el alcance y algunos efectos del programa Becas 6000 en Andalucía". Pp. 501514 en La educación en la sociedad global e informacional. XVI Conferencia de Sociología de la Educación, editado por Xavier Bonal, Capitolina Díaz, y David Luque. Oviedo: Asociación de Sociología de la Educación.

Río Ruiz, M.A. 2014. "Efectos de la conversión en becario y consecuencias de la reforma del sistema de becas entre universitarios de clase obrera". RASE, Revista de la Asociación de la Sociología de la Educación 7: 468-487.

San Segundo, M. J. 2005. "Política de becas y ayudas al estudio en los presupuestos del año 2005". Presupuesto y Gasto Público 38: 7-30.

Slavin, R. E. 2010. "Can Financial Incentives Enhance Educational Outcomes? Evidence from International Experiments". Educational Research Review 5:68-80. 
Terrail, J. P. 1984. "Familles ouvrières, école, destin social (1880-1980)". Revue Française de Sociologie 25: 421-36.

Valencia, E. 2008. "Conditional Cash Transfers as Social Policy in Latin America: an Assessment of their Contributions and Limitations". Annual Review of Sociology 34: 475-99.

Manuel Ángel Río Ruiz es Doctor en Sociología y premio extraordinario de doctorado por la Universidad de Sevilla. Es profesor contratado doctor en el Departamento de Sociología de la Universidad de Sevilla. Coordinador del grupo de investigación "Dominación y Desigualdades Sociales en Andalucia". Principales de líneas de investigación: Políticas y desigualdades educativas, exclusión social y etnicismo.

María LUISA JimÉnez Rodrigo es Doctora en Antropología Social por la Universidad de Granada. Es profesora contratada doctora en el Departamento de Sociología de la Universidad de Sevilla. Principales líneas de investigación: Políticas sociolaborales, desigualdades de género y sociología de la salud.

RECIBIDO: 05/03/2013

ACEPTADO: 16/12/2013

PUBLICADO ONLINE: 22/07/2014 